\title{
Genotype-by-sex-by-diet interactions for nutritional preference, dietary consumption, and lipid deposition in a field cricket
}

\author{
James Rapkin ${ }^{1} \cdot$ Kim Jensen ${ }^{2} \cdot$ Clarissa M. House ${ }^{1,3,4} \cdot$ Alastair J. Wilson $^{1} \cdot$ John Hunt $^{1,3,4}$
}

Received: 14 March 2018 / Revised: 5 July 2018 / Accepted: 14 July 2018 / Published online: 8 August 2018

(c) The Genetics Society 2018

\begin{abstract}
Changes in feeding behaviour, especially the overconsumption of calories, has led to a rise in the rates of obesity, diabetes, and other associated disorders in humans and a range of animals inhabiting human-influenced environments. However, understanding the relative contribution of genes, the nutritional environment, and their interaction to dietary intake and lipid deposition in the sexes still remains a major challenge. By combining nutritional geometry with quantitative genetics, we determined the effect of genes, the nutritional environment, and their interaction on the total nutritional preference (TP), total diet eaten (TE), and lipid mass (LM) of male and female black field crickets (Teleogryllus commodus) fed one of four diet pairs (DPs) differing in the ratio of protein to carbohydrate and total nutritional content. We found abundant additive genetic variance for TP, TE, and LM in both sexes and across all four DPs, with significant genetic correlations between TE and TP and between TP and LM in males. We also found significant genotype-by-DP and genotype-by-sex-by-DP interactions for each trait and significant genotype-by-sex interactions for TE and LM. Complex interactions between genes, sex, and the nutritional environment, therefore, play an important role in nutrient regulation and lipid deposition in $T$. commodus. This finding may also help explain the increasing rate of obesity and the maintenance of sex differences in obesity observed across many animal species, including humans.
\end{abstract}

\section{Introduction}

The overconsumption of calories has been associated with the rise in worldwide rates of obesity, cardiovascular disease, diabetes, and other disorders and diseases in humans and a range of animal species associated with humans in industrialized societies (Klimentidis et al. 2011;

Electronic supplementary material The online version of this article (https://doi.org/10.1038/s41437-018-0130-x) contains supplementary material, which is available to authorized users.

John Hunt

J.Hunt@westernsydney.edu.au

1 Centre for Ecology and Conservation, University of Exeter, Cornwall Campus, Penryn, Cornwall TR10 9FE, UK

2 Department of Bioscience, Terrestrial Ecology, Aarhus University, Vejlsøvej 25, 8600 Silkeborg, Denmark

3 School of Science and Health, Western Sydney University, Locked Bag 1797, Penrith, NSW 2751, Australia

4 Hawkesbury Institute for the Environment, University of Western Sydney, Locked Bay 1797, Penrith, NSW 2751, Australia
Raubenheimer et al. 2015). This overconsumption is puzzling because optimal foraging theory predicts that animals should evolve regulatory foraging mechanisms to optimize their evolutionary fitness (Stephens and Krebs 1986; Simpson and Raubenheimer 2012). Traditionally, theory has assumed that optimizing fitness required maximizing energy intake (Stephens and Krebs 1986). However, more recent developments using nutritional geometry have found that optimizing fitness requires animals to regulate both their energy intake and the specific balance (or ratio) of nutrients (Simpson and Raubenheimer 2012).

Nutritional geometry is a multidimensional nutritional framework that can be used to quantify the independent and interactive effects of nutrient intake on phenotypic traits and determine how individuals prioritize the intake of these nutrients (Simpson and Raubenheimer 2012). The effect of nutrient intake on a given trait is measured by restricting a series of individuals to a single diet taken from a larger array of diets that vary in nutrient ratio and concentration (Simpson and Raubenheimer 2012). Nutrient intake and the phenotypic trait(s) is then precisely measured for all individuals and the relationship quantified statistically using response surface methodologies and visualized by 
constructing nutritional landscapes (South et al. 2011). Determining how individuals prioritize the intake of nutrients is typically achieved by presenting individuals with the choice between alternate pairs of diets differing in nutrient ratio and concentration (Simpson and Raubenheimer 2012). The average intake of nutrients across diet pairs (DPs) is referred to as the regulated intake point (RIP) (or target) and reflects the point in nutrient space that individuals defend when given dietary choice (Simpson and Raubenheimer 2012). If this RIP is in close proximity or resides entirely on the peak of the trait when mapped onto the nutritional landscape, nutrient regulation is considered optimal for the expression of this trait (Rapkin et al. 2018). Examples of active nutrient regulation can be found in a diversity of insect species, including predatory ground beetles (Anchomenus dorsalis; Jensen et al. 2015), fruit flies (Drosophila melanogaster; Lee et al. 2008; Jensen et al. 2015), speckled roaches (Nauphoeta cinerea; South et al. 2011; Bunning et al. 2015, 2016), and field crickets (Teleogryllus commodus; Maklakov et al. 2008; Rapkin et al. 2017; Gryllus veletis, Harrison et al. 2014). However, with the exception A. dorsalis, nutrient regulation in these species does not appear optimal for the expression of important phenotypic traits, such as reproduction (Lee et al. 2008; Maklakov et al. 2008; South et al. 2011; Bunning et al. 2015, 2016; Jensen et al. 2015; Rapkin et al. 2017) and lifespan (Lee et al. 2008; Maklakov et al. 2008; Jensen et al. 2015; Rapkin et al. 2017). As traits frequently have different nutritional optima (e.g., Lee et al. 2008; Maklakov et al. 2008; Jensen et al. 2015), it has been argued that this sub-optimal nutrient regulation may represent an attempt by individuals to balance the expression of multiple traits (Lihoreau et al. 2015; Bunning et al. 2016). Alternatively, sub-optimal regulation may reflect a constraint on optimal feeding behaviour mediated through inefficiencies in dietary assimilation, digestion, absorption, and/or utilization (Henson and Hallam 1995).

An important finding from studies using nutritional geometry is that many animal species have separate appetite systems for the intake of macronutrients (protein, carbohydrate, and fat) and that equal priority is not always given to each system (Raubenheimer and Simpson 1997; Gosby et al. 2014). When restricted to a diet of fixed macronutrient intake, the intake of protein is often more strongly regulated than carbohydrate and/or fat; this asymmetry has been termed the protein leverage hypothesis (PLH) (Simpson and Raubenheimer 2005; Sørensen et al. 2008). According to the PLH, when the proportion of protein in a diet is reduced, the more powerful protein appetite stimulates an increased consumption of diet to gain more of the limited supply of this nutrient. As a result, diets that are higher in carbohydrate and/or fat that dilute the availability of protein will promote increased consumption and overall intake of energy (Simpson and Raubenheimer 2005). It has therefore been argued that the PLH can explain the rise in levels of obesity and disease because of a shift towards consuming energy-dense foods that are high in carbohydrates and/or fats but low in protein (Brooks et al. 2010; Gosby et al. 2014; Raubenheimer et al. 2015). Empirical support for the PLH comes from studies on spider monkeys (Ateles chamek; Felton et al. 2009), humans (Gosby et al. 2011, 2014; Martens et al. 2013), and mice (Sørensen et al. 2008), although a direct link between feeding behaviour and obesity in these species in less concrete.

Despite the many key insights provided by nutritional geometry, most studies using this approach to examine nutrient regulation have focussed on the RIP and have largely ignored the potential importance of variation in intake that exists around this point (Simpson and Raubenheimer 2012). One source of variation in nutrient intake that is likely to be important is the genotype of an individual. Indeed, studies on humans (e.g., Tanaka 2014), rats (e.g., Liu and Lloyd 2013), mice (e.g., Smith et al. 2000), fruit flies (Reddiex et al. 2013), and field crickets (Rapkin et al. 2017) all indicate that the intake of macronutrients has a genetic basis. The predisposition to the deleterious effects of dietary overconsumption also appears to have a genetic basis that varies with the nutritional environment (van der Klaauw and Farooqi 2015), with genotype-by-diet interactions for weight gain and obesity being demonstrated in mice (Sutton et al. 2006; Gordon et al. 2008), D. melanogaster (Reed et al. 2010), and humans (Qi and Cho 2008; Heianza and Qi 2017). While there is less support, there is some evidence in humans to suggest that the genes for dietary intake are linked to those for obesity (e.g., Faith et al. 1999; Hasselbalch et al. 2010). For example, twinstudies have identified positive genetic correlations between the intake of calories and both body mass (Faith et al. 1999) and body fat (Hasselbalch et al. 2010), although the latter association was found in men but not women. This suggests that dietary intake and obesity are unlikely to evolve as independent traits (Lande 1979), although clearly more empirical work is needed on non-human species to verify this.

In the majority of sexually reproducing species, males and females share most of their genomes and express many of the same traits (Lande 1980). Consequently, shared traits, such as dietary intake and obesity, are often genetically correlated between the sexes. However, it is unlikely that the genetic basis of these traits, as well as how the genes for these traits interact with the nutritional environment, will be constant across the sexes. This is because the sexes often have different nutritional requirements for optimal reproduction (Simpson and Raubenheimer 2012). For example, in D. melanogaster (Jensen et al. 2015) and two species of field cricket (T. commodus, Maklakov et al. 2008; Rapkin 
et al. 2017; G. veletis, Harrison et al. 2014), female reproduction is maximized at a high consumption of both protein and carbohydrate in an equal 1:1 ratio of protein to carbohydrate, whereas a relatively higher intake of carbohydrate is needed to maximize male reproduction, with males performing better on diets with a higher carbohydrate to protein ratio. Furthermore, there is often sex differences in both the incidence (e.g., Klimentidis et al. 2011; Link and Reue 2017) and deleterious effects of obesity (e.g., CoatmellecTaglioni et al. 2003; Song et al. 2016), as well as the effects of dietary intake on obesity (e.g., Medrikova et al. 2012; Yuan et al. 2016). For example, in many human populations the incidence of obesity and associated metabolic diseases is higher in men than women (reviewed in Link and Reue 2017) and dietary intake often has very different effects on obesity in the sexes (e.g., Yuan et al. 2016). As these differences in nutrient intake and obesity have important fitness consequences, they will be subject to differential patterns of selection in the sexes that are likely to shape the underlying genetic architecture of these traits (Lande 1980). This includes differences in the genetic variances of nutrient intake and obesity in the sexes, as well as differences in the genetic covariance between these traits within and across the sexes, that are likely to result in a complex pattern of genotype-by-sex and/or genotype-by-diet-by-sex interactions for these traits. Despite the potential importance to how nutrient intake and obesity evolve, surprisingly few studies have formally quantified these interactions.

Here we combine nutritional geometry with quantitative genetics to determine how male and female black field crickets ( $T$. commodus) of known genetic relatedness respond when placed into four different nutritionally imbalanced environments varying in both the ratio and concentration of protein (P) and carbohydrate (C). If individuals actively regulate nutrient intake, we predict that there will be differences in the total amount of diet eaten and the total nutrient preference across differing DPs as individuals adjust not only their intake of nutrients but also the ratio of protein to carbohydrate they ingest to maintain, as close as possible, a preferred nutrient intake, which will influence overall lipid deposition. Moreover, if males and females differentially regulate nutrient intake, we predict that the total amount of diet eaten and total nutrient preference will differ across the sexes, as will the relationship between these traits and lipid deposition. Finally, if nutrient regulation and lipid deposition are under genetic control and these traits are genetically associated, we predict significant additive genetic variance in these traits and additive genetic covariances between these traits within each sex and DP. However, we also predict that any differences in these genetic parameters between the sexes and across DPs will result in complex interactions between genotype, DP, and sex for the total amount of diet eaten, total nutrient preference, and lipid deposition. Such interactions would indicate these traits are not free to evolve independently across DPs and the sexes and therefore may help explain why rates of obesity are increasing on a global scale and why sex differences in obesity exist in many species, including humans (Klimentidis et al. 2011; Raubenheimer et al. 2015).

\section{Materials and methods}

\section{Study species}

A total of 200 mated female T. commodus were collected from Smith's Lake, New South Wales in eastern Australia in March 2009 and used to establish a large panmictic lab population, maintained in 10 large culture containers $(100 \mathrm{~L})$ of approximately 500 animals per culture for 10 non-overlapping generations prior to this experiment. Lab populations are kept at $28 \pm 1{ }^{\circ} \mathrm{C}$, under a 13:11 light:dark cycle, cleaned weekly and provided with cardboard egg cartons for shelter, water ab libitum, egg pads consisting of damp cotton wool in a petri dish and a mixture of cat food (Purina Go Cat Senior ${ }^{\odot}$, St. Louis, MO, USA), and rat food (SDS Diets, Essex, UK). Nymphs were moved at random between culture containers each generation to ensure gene flow. While $T$. commodus is wing polymorphic, the longwinged morph is only rarely observed at the collection locality and has never been observed in our laboratory population.

\section{Artificial diets}

Using the protocol established in South et al. (2011), we made four powdered, holidic (i.e., chemically defined) diets. These four diets were used to make four dietary pairs, with each pair containing one diet with a P:C ratio of $1: 8$ and one with a P:C ratio of $5: 1$. We provided these diets in one of two nutritional dilutions ( $\% \mathrm{P}+\mathrm{C}$ content), 36 or $84 \%$. The four DPs are as follows: DP1: 1:8 (36\%) vs. 5:1 (36\%), DP2: 1:8 (84\%) vs. 5:1 (36\%), DP3: 1:8 (36\%) vs. 5:1 (84\%), and DP4: 1:8 (84\%) vs. 5:1 (84\%) with composition also provided in Table S1. Diets were selected from a larger geometric array of 24 diets because they provide a broad coverage of potential nutrient space (Fig. S1) and have been used in previous choice feeding experiments (South et al. 2011; Bunning et al. 2015).

\section{Quantitative genetic breeding design}

To estimate the quantitative genetics of total diet eaten, nutrient preference, and lipid mass, we used a split-brood half-sib breeding design whereby sons and daughters from 
each full-sib family were split across four different DPs and their intake of nutrients measured under dietary choice for 21 days. The half-sib breeding design was established by mating each of 30 randomly chosen virgin sires with three randomly chosen dams. A total of 50 offspring from each dam were collected and reared in a family group in an individual plastic container $(10 \times 10 \times 5 \mathrm{~cm})$ for 3 weeks, with access to an ad libitum supply of ground cat food (Purina Go Cat Senior ${ }^{\odot}$, St. Louis, MO, USA) and water provided in a $5 \mathrm{~cm}$ plastic tube plugged with cotton wool. After 3 weeks, 12 sons and 12 daughters per dam were isolated and established at random in individual plastic containers $(5 \mathrm{~cm} \times 5 \mathrm{~cm} \times 5 \mathrm{~cm})$ and provided with ad libitum cat food pellets and water, and checked daily for eclosion to adulthood. Containers were cleaned and fresh food and water were provided weekly.

On the day of eclosion, we randomly allocated three sons and three daughters per dam to each of four DPs (total $n=$ 1080 sons and 1080 daughters; see Fig. S2 for a schematic representation of our breeding design). Fresh diet was provided every 3 days (i.e., a total of seven feeding periods). Experimental animals were mated with a stock animal of the opposite sex on the evening of day 8 post-eclosion and removed on day 9 with females provided with a petri dish of moist sand thereafter for oviposition.

\section{Feeding regime}

Experimental feeding followed our established protocols (South et al. 2011). In brief, two dishes of diet of measured dry weight were provided to each cricket according to assigned DP. Food was provided in feeding platforms constructed by gluing the upturned lid of a vial $(1.6 \mathrm{~cm}$ diameter, $1.6 \mathrm{~cm}$ deep) onto the middle of a petri dish $(5.5 \mathrm{~cm}$ diameter) and water was provided ad libitum in a $5 \mathrm{ml}$ test tube plugged with cotton wool. Any diet spilled during feeding was collected in the petri dish and weighed. All diets were dried in an oven (Binder FD115, Germany) at $30{ }^{\circ} \mathrm{C}$ for $72 \mathrm{~h}$ before weighing. Feeding platforms were weighed before and after each feeding period using an electronic balance (Ohaus Explorer Professional EP214C, Switzerland). Faeces were removed from the diet and feeding platform using forceps prior to re-weighing. Diet consumption was calculated as the difference in dry weight of diet before and after feeding. This amount of consumed diet was converted to a weight of $\mathrm{P}$ and $\mathrm{C}$ ingested by multiplying by the proportion of these nutrients in the diet (South et al. 2011).

\section{Measuring lipid mass}

On day 21 , crickets were frozen and stored at $-20^{\circ} \mathrm{C}$ and lipid extraction was performed using the protocol outlined in South et al. (2011). In brief, each cricket was defrosted to room temperature and a slit was made along the abdomen using dissecting scissors. The cricket was then dried at $60{ }^{\circ} \mathrm{C}$ for $24 \mathrm{~h}$ and weighed using an electronic balance. Each cricket was then placed in $10 \mathrm{ml}$ of a 2:1 (v/v) solution of dichloromethane:methanol and agitated for $48 \mathrm{~h}$ to extract lipids. Crickets were then removed from this solution and dried for a further $24 \mathrm{~h}$ at $60^{\circ} \mathrm{C}$ and then weighed. The difference between the pre-extraction and postextraction weights was taken as the lipid mass.

\section{Statistical analysis}

Quantitative genetic analyses were performed using animal models fitted in ASReml (version 3) (Gilmour et al. 2009). An animal model is a form of linear mixed-effect model where an individual's genetic pedigree is included as a random effect allowing for the estimation of the additive genetic (co)variance for phenotypic traits (Wilson et al. 2010). We examined three phenotypic traits: the total amount of diet eaten (TE) (including nutritional and nonnutritional components), total nutritional preference (TP) (calculated as total protein intake divided by total carbohydrate intake, where higher values indicate a bias towards protein intake), and total body lipid mass (LM) (as a measure of fat deposition). Prior to analysis each trait was standardized to a mean of zero and standard error of one using a $Z$-transformation and body size (measured as pronotum width) was included as a fixed effect in all models to control for any size effects on TE, TP, or LM.

We first tested for the effect of sex and DP on our three traits using Wald- $F$ tests. Given the significant effect of sex and DP on all three traits (see Results), we included these as fixed effects in a univariate model and estimated the additive genetic variance $\left(V_{\mathrm{A}}\right)$ for each trait by comparing univariate models run without and with the addition of the breeding values as a random effect for each trait. We then examined the presence and strength of any interactions between the genotype $(\mathrm{G})$ and the dietary environment and between $\mathrm{G}$ and sex. We tested for a G-by-DP interaction by running univariate models for each trait but split across the four DPs with sex included as a fixed effect. Similarly, we tested for a G-by-Sex interaction by running univariate models for each trait but split across the sexes with DP included as a fixed effect. In both cases, a secondary analysis was performed to explore sex and DP differences by restricting G-by-DP models to one sex at a time and restricting G-by-Sex models to one DP at a time. Finally, we tested for G-by-Sex-by-DP interactions by running univariate models for each trait split across each DP for males and females.

We also extracted estimates of additive genetic (co)variances, heritabilities $\left(h^{2}\right)$, and genetic correlations $\left(r_{\mathrm{A}}\right)$ from 
these models (Table 3 ). These estimates were presented in a matrix form, commonly referred to as a genetic (co)variance matrix (or commonly referred to as a $\mathrm{G}$ matrix), containing the additive genetic variances along the diagonal, additive genetic covariances below the diagonal, and genetic correlations above the diagonal (Table 3). Model summaries and log-likelihoods for all our quantitative genetic models can be found in Tables S2 and S3 and example ASReml code can be found in Text S1. All statistical inference was based on likelihood-ratio tests (LRT). Due to the greater mathematical complexity in fitting multivariate models with an increasing number of response variables, we were unable to run a single multivariate (multi-trait) model which included each trait split by sex and DP treatments (e.g., 3 Traits $\times 2$ Sexes $\times 4$ DPs $=24$ Trait $\times$ Sex $\times$ DP combinations).

Finally, given the difference in TE, TP, and LM across DPs and the sexes (see Results) we also explored the effects of $\mathrm{P}$ and $\mathrm{C}$ intake on LM and whether this differed across the sexes. We used a response surface approach to characterize the linear and non-linear (quadratic and correlational) effects of nutrients on LM in each sex (South et al. 2011). We visualized the effects of $P$ and $C$ intake on $L M$ in each sex using thin-plate splines constructed using the Tps function in the FIELDS package of $\mathrm{R}$ (version 2.15.1, www.r-project.org). We then statistically compared the linear, quadratic, and correlational effects of nutrient intake across the sexes using a sequential model building approach outlined in South et al. (2011).

\section{Results}

There was a significant effect of DP and sex on the total amount of diet eaten (TE) (including nutritional and nonnutritional components), total nutritional preference (TP) (calculated as total protein intake divided by total carbohydrate intake), and total body lipid mass (LM) (as a measure of fat deposition) (Table 1). For both sexes, TE was highest on DP1, followed by DP3, DP2, and lowest on DP4 which is consistent with compensatory feeding in the sexes. Males and females increased their consumption of diet by 58 and $72 \%$, respectively, when feeding on the lowest (DP1, 36\% nutrition) vs. the highest (DP4, 84\% nutrition) nutrient DP indicative of compensatory feeding. Females consumed more diet than males on each DP and their consumption of diets was, on average, 20\% higher than males across all DPs (Fig. S3).

TP was highest for DP3 in both sexes, which indicates a strong bias towards protein consumption, followed by DP1, DP4, and DP2 with TP values being greater for females than males on each DP. This can be visualized in Fig. 1, which shows the mean $\mathrm{P}$ and $\mathrm{C}$ intake of the sexes on each DP, as well as the RIP for each sex (calculated as the mean
Table $1 F$-tests examining the significance of body size, sex, and diet pair on our three trait measures: total eaten, total preference, and lipid mass

\begin{tabular}{llll}
\hline & $F$ & df & $P$ \\
\hline Total eaten & & & \\
Sex & 407.92 & 1,2154 & 0.001 \\
Diet pair & 527.45 & 3,2154 & 0.001 \\
Total preference & & & \\
Sex & 2035.36 & 1,2154 & 0.001 \\
Diet pair & 437.44 & 3,2154 & 0.001 \\
Lipid mass & & & \\
Sex & 272.48 & 1,2154 & 0.001 \\
Diet pair & 238.75 & 3,2154 & 0.001 \\
\hline
\end{tabular}

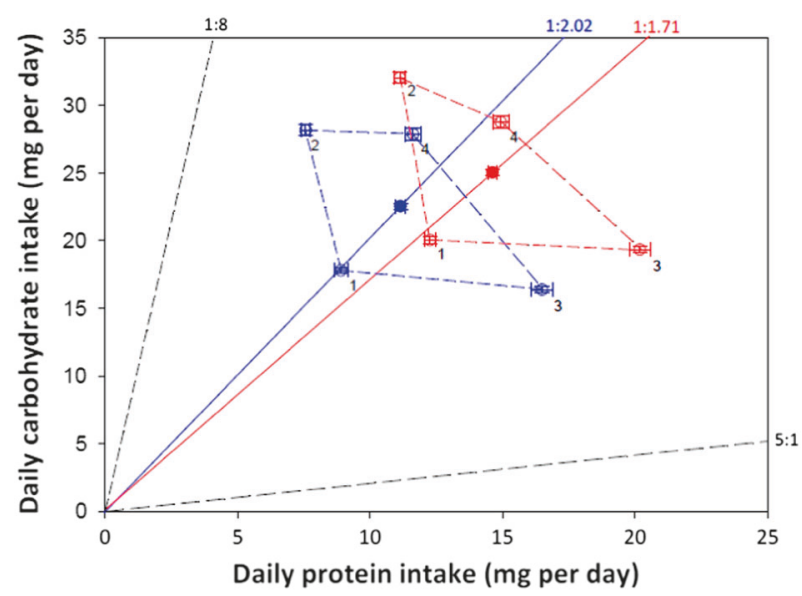

Fig. 1 The mean $( \pm \mathrm{SE})$ intake of $\mathrm{P}$ and $\mathrm{C}$ by male (blue symbols) and female (red symbols) T. commodus. The open symbols represent the mean intake of nutrients in each of the four diet pairs (denoted by pair number), whereas the solid symbols represent the regulated intake point (RIP), calculated as the mean of the four diet pairs. The solid blue and red lines represent the nutritional rails (lines in nutrient space that represents a fixed intake of nutrients) that passes through the RIP for males (P:C ratio of 1:2.02) and females (P:C ratio of 1:1.71). The black dashed lines (P:C ratios of 5:1 and 1:8) represent the outer nutritional rails of the nutritional landscape

intake of these nutrients across DPs and represents the point in nutrient space that individuals actively defend when given dietary choice). With the exception of DP3, crickets on all other DPs showed a preference to consume relatively more $\mathrm{C}$ than $\mathrm{P}$ (Fig. 1). However, this $\mathrm{C}$ biased preference was more prominent in males with a RIP at a $\mathrm{P}: \mathrm{C}$ ratio of 1:2.02 than females with a RIP at a P:C ratio of 1:1.71 (Fig. 1), with non-random feeding, confirming active nutrient regulation, found for both sexes in all four DPs (Fig. S4).

For both sexes LM was highest on DP4, followed by DP2, DP3, and DP1 (Fig. 2). However, despite the higher consumption of diets by females, LM was actually higher in 
Fig. 2 Thin-plate spline (contour view) visualizations of the effects of protein $(\mathrm{P})$ and carbohydrate (C) intake on lipid mass in a female and $\mathbf{b}$ male Teleogryllus commodus. In each spline, the red regions represent higher values for the measured trait, whereas blue regions represent lower values. The white crosses represent the RIPs from Fig. 1 overlaid on the respective female and male landscapes. The black symbols represent the mean $\mathrm{P}$ and $\mathrm{C}$ intake of each sire within the four diet pairs
A

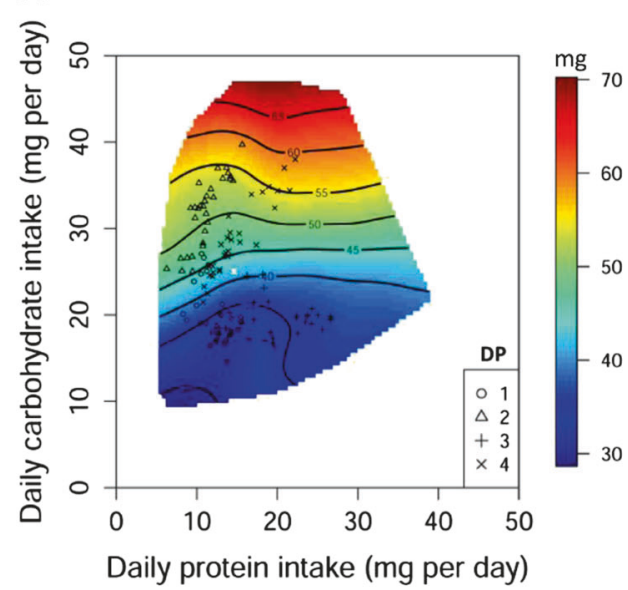

B

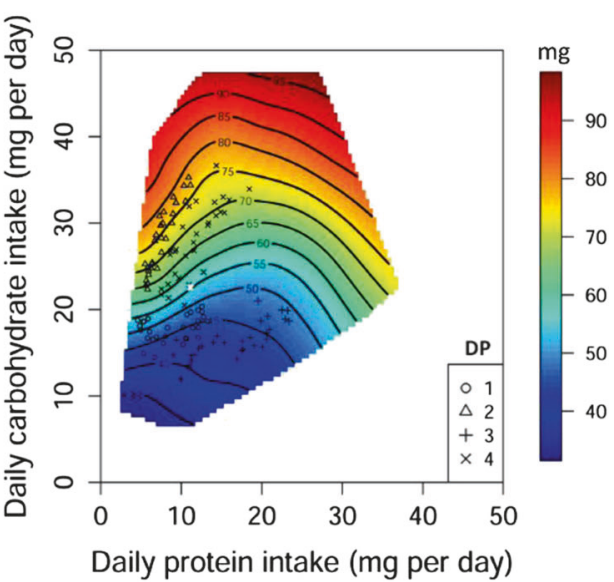

Table 2 Response surface analysis quantifying the linear and nonlinear effects of protein $(\mathrm{P})$ and carbohydrate $(\mathrm{C})$ intake on lipid deposition in male and female Teleogryllus commodus

\begin{tabular}{lllllll}
\hline & \multicolumn{2}{l}{ Linear effects } & & \multicolumn{2}{l}{ Non-linear effects } \\
\cline { 2 - 3 } Sex & $\mathrm{P}$ & $\mathrm{C}$ & & $\mathrm{P} \times \mathrm{P}$ & $\mathrm{C} \times \mathrm{C}$ & $\mathrm{P} \times \mathrm{C}$ \\
\hline Males & & & & & \\
Gradient & $\mathbf{- 0 . 0 8} \pm$ & $\mathbf{0 . 5 2} \pm$ & & $\mathbf{0 . 0 9} \pm$ & $-0.00 \pm$ & $-\mathbf{0 . 1 0} \pm$ \\
\pm SE & $\mathbf{0 . 0 3}$ & $\mathbf{0 . 0 3}$ & & $\mathbf{0 . 0 2}$ & 0.02 & $\mathbf{0 . 0 3}$ \\
$t_{1029}$ & $\mathbf{3 . 1 4}$ & $\mathbf{1 9 . 3 8}$ & $\mathbf{4 . 2 3}$ & 0.06 & $\mathbf{2 . 8 7}$ \\
$P$ & $\mathbf{0 . 0 0 2}$ & $\mathbf{0 . 0 0 0 1}$ & $\mathbf{0 . 0 0 0 1}$ & 0.95 & $\mathbf{0 . 0 0 4}$ \\
Females & & & & & \\
Gradient & $-0.03 \pm$ & $\mathbf{0 . 4 9} \pm$ & $\mathbf{0 . 0 4} \pm$ & $0.02 \pm$ & $-0.00 \pm$ \\
\pm SE & 0.03 & $\mathbf{0 . 0 3}$ & $\mathbf{0 . 0 2}$ & 0.03 & 0.03 \\
$t_{1041}$ & 1.09 & $\mathbf{1 8 . 1 2}$ & $\mathbf{2 . 0 0}$ & 0.81 & 0.07 \\
$P$ & 0.27 & $\mathbf{0 . 0 0 0 1}$ & $\mathbf{0 . 0 4}$ & 0.42 & 0.95 \\
\hline
\end{tabular}

Significant $(P<0.05)$ linear and non-linear effects are highlighted in bold

males (Fig. 2). Response surface analysis showed that LM increased linearly with the intake of $\mathrm{C}$ in both sexes and decreased linearly with $\mathrm{P}$ intake in males but not in females (Table 2). There were significant positive quadratic effects of $\mathrm{P}$ intake on LM in both sexes but no significant quadratic effects of $\mathrm{C}$ intake (Table 2). There was a significant negative correlational effect of nutrient intake on LM in males but not females (Table 2). The effect of nutrient intake on LM in the sexes is presented as thin-plate splines in Fig. 2 and they confirm that LM is maximized at a high intake of $\mathrm{C}$ and low intake of $\mathrm{P}$ in both sexes. Indeed, a sequential model-building approach revealed that linear $\left(F_{2,2068}=1.16, P=0.31\right)$, quadratic $\left(F_{2,2064}=2.33, P=\right.$ $0.10)$, and correlational $\left(F_{1,2062}=2.80, P=0.10\right)$ effects of $\mathrm{P}$ and $\mathrm{C}$ intake on $\mathrm{LM}$ did not differ significantly between the sexes.
LRT tests found significant additive genetic variance for TE, TP, and LM in each sex and across the four DPs (Models A-B, Table S2). We also found evidence for significant G-by-DP interactions for each trait with a univariate model containing just $G$ significantly improved with the addition of a G-by-DP interaction term (Models C-D, Table S3). Further exploration within each sex showed that this interaction was significant for all three traits in both males and females, being especially pronounced for TP (Table S4). These interactions can be visualized in the reaction norms provided in Fig. 3, with multiple crossovers signalling that different genotypes respond differently across DP, indicative of significant G-by-DP interactions. We also found evidence for significant G-by-Sex for TE and LM but not TP with univariate models for TE and LM significantly improved by the addition of a G-by-Sex interaction term (Models E-F, Table S3). Further exploration within each DP showed that this interaction was significant in all four DPs for TE and LM but was only significant in DPs 1, 2, and 3 for TP (Table S4). These interactions can be visualized in the reaction norms provided in Fig. 4 with multiple crossovers signalling significant G-by-Sex interactions for each trait but more so for TE and LM than TP, especially in DP4.

Finally, we found evidence for significant G-by-Sex-byDP interaction for TE, TP, and LM with the fit of univariate models being significantly improved by the addition of this interaction term (Models G-H, Table S3). This finding suggests that complex interactions between genes, sex, and the nutritional environment are key to the intake of nutrients and lipid deposition in male and female T. commodus. More specifically, it indicates that individuals are genetically predisposed to regulate their nutrient intake or deposit lipid but this depends on variation in the nutritional environment and their sex. A significant G-by-Sex-by-DP interaction also suggests that the additive genetic variance-covariance structure among these traits is also likely to change 


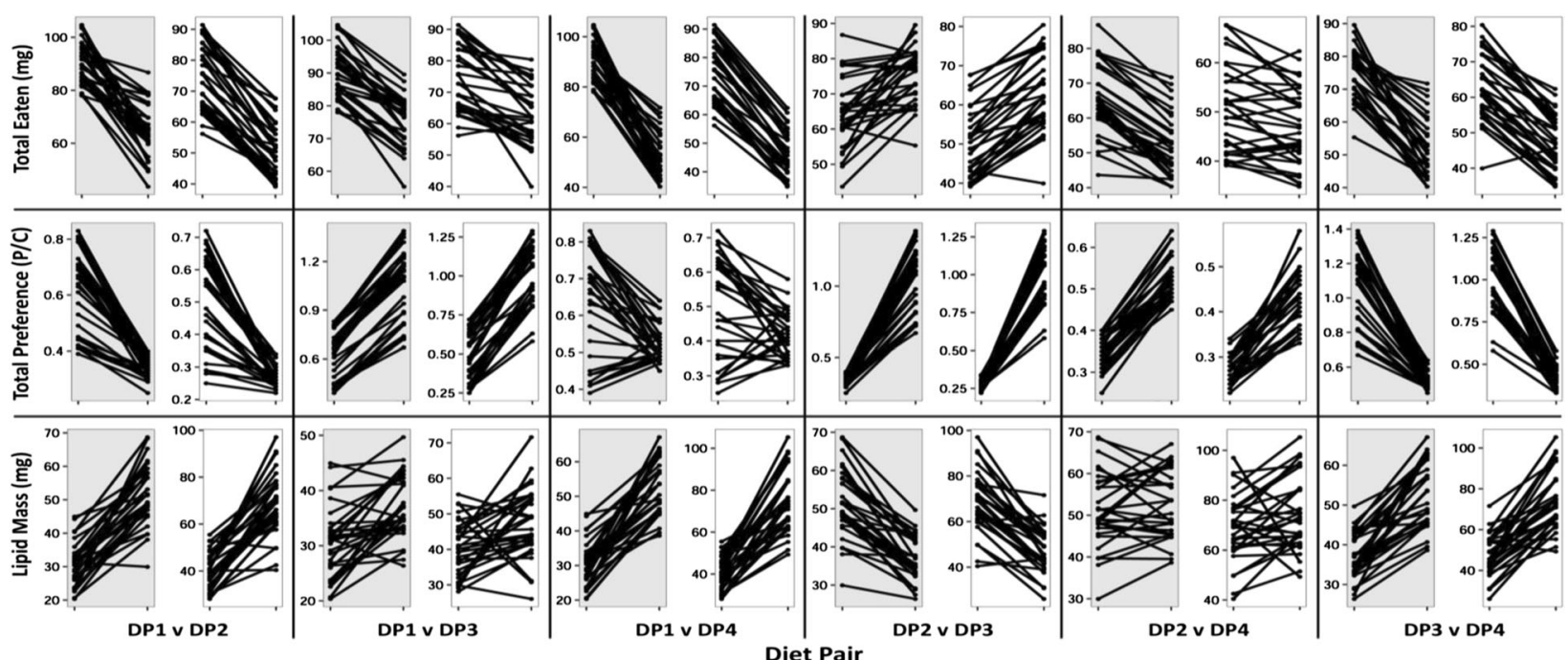

Fig. 3 Reactions norms illustrating the genotype-by-diet pair interaction (G-by-Sex) for the total amount of diet eaten (TE), total nutrient preference (TP), and lipid mass (LM) in male and female T. commodus. Females are presented with a grey background and males with a white background. Each column of the figure presents a specific diet pair comparison between the sexes for each trait. In each panel, lines represent the response of a given genotype across two diet pairs

commodus. We provide clear evidence of sex-specific nutrient intake in this species and evidence of ample additive genetic variance in TE, TP, and LM in both sexes and across all DPs (the only exception being for TP in females for DP4). Most importantly, we provide evidence for significant G-by-DP and G-by-Sex-by-DP interactions for each trait, as well as significant G-by-Sex interactions for TE and LM but not for TP. Our findings demonstrate that complex interactions between genotype, sex, and the nutritional environment play a central role in how $T$. commodus regulate their feeding behaviour and may help explain some general patterns observed for obesity across species. For example, the high $h^{2}$ estimates for TE, TP, and LM and the genetic correlations between these traits show that there is a high potential for these traits to evolve but they are unlikely to do so independently. This may explain the increasing rate of obesity observed in numerous species (Klimentidis et al. 2011; Raubenheimer et al. 2015) as LM will not only evolve directly but also indirectly through any evolutionary changes in feeding behaviour (Lande 1980). Moreover, the existence of G-by-Sex and G-by-DP interactions may explain why there are often sex differences in the incidence of obesity (Kanter and Caballero 2012) and the rate of increase in obesity over time across species (Klimentidis et al. 2011). However, before the generality of these patterns can be confirmed, more quantitative genetic studies are needed across a wider range of species.

Optimal foraging theory (Stephens and Krebs 1986) predicts that when in a nutritionally imbalanced environment, an animal may actively regulate their intake of nutrients either through compensatory feeding or by eating non-randomly from multiple food sources (Simpson and (TP), and total body lipid mass (LM) in male and female $T$. 

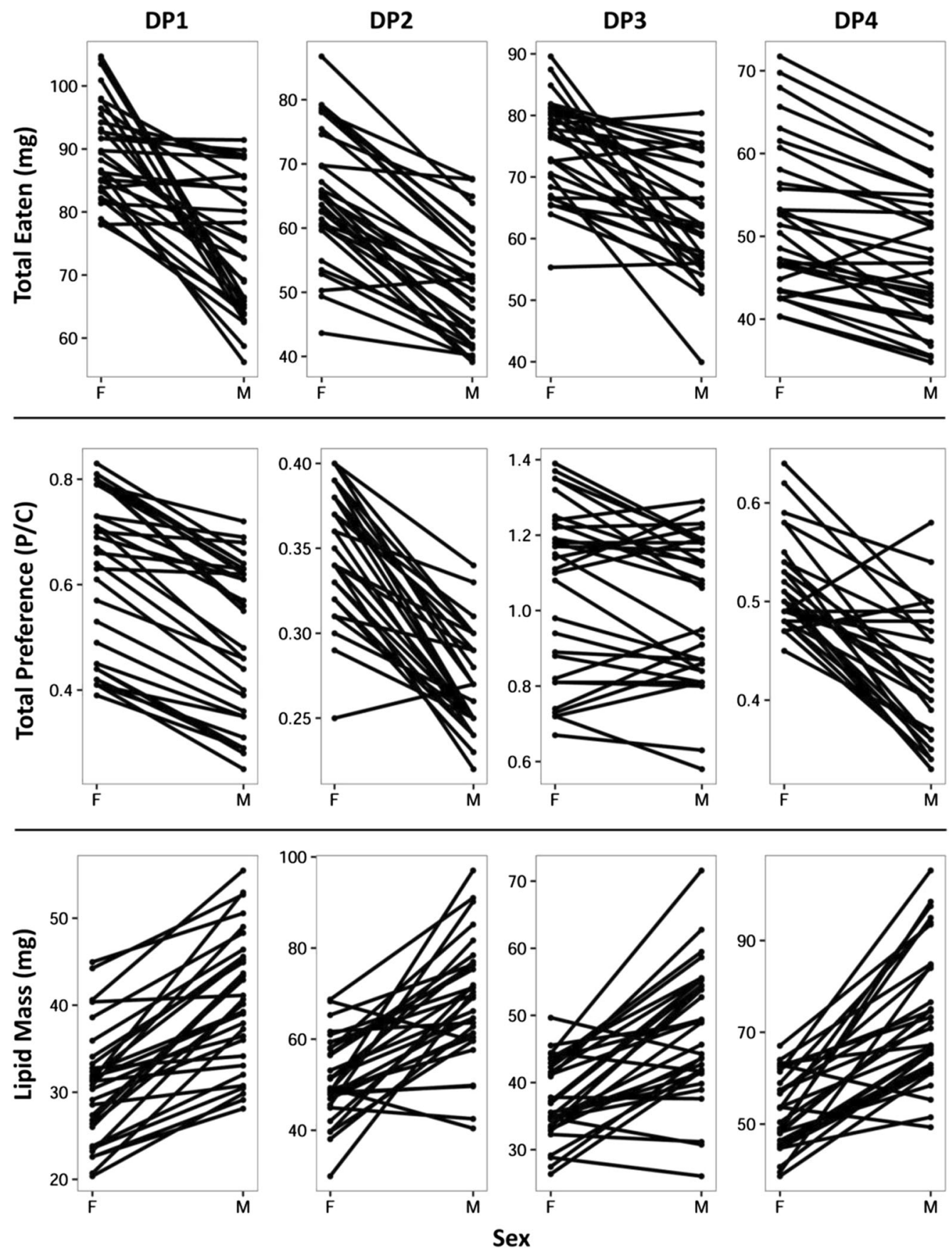

Fig. 4 Reaction norms illustrating the genotype-by-sex interaction (G-by-Sex) for the total eaten (TE), the total nutritional preference (TP), and lipid mass (LM) in the different diet pairs in T. commodus. In each panel, lines represent the response of a given genotype across two diet pairs

Raubenheimer 2012). Our finding that there is considerable variation in both TE and TP across DPs and the sexes suggests that both processes are operating in male and female $T$. commodus but to differing degrees. We found that both sexes increased the total amount of diet they consumed on the lowest nutrition pair (DP1) compared to highest nutrition pair (DP4) but this increase was larger in females (72\%) than males (52\%). While compensatory feeding has been demonstrated in a variety of animal taxa (Simpson and Raubenheimer 2012), only a single study on Nile tilapia (Oreochromis niloticus) has found sex differences in this behaviour (Barreto et al. 2003). In contrast to our work, however, compensatory feeding is more pronounced in males than females in this species (Barreto et al. 2003). We also show that females have consistently higher TP values than males on each DP with the RIP being relatively more $\mathrm{P}$ 


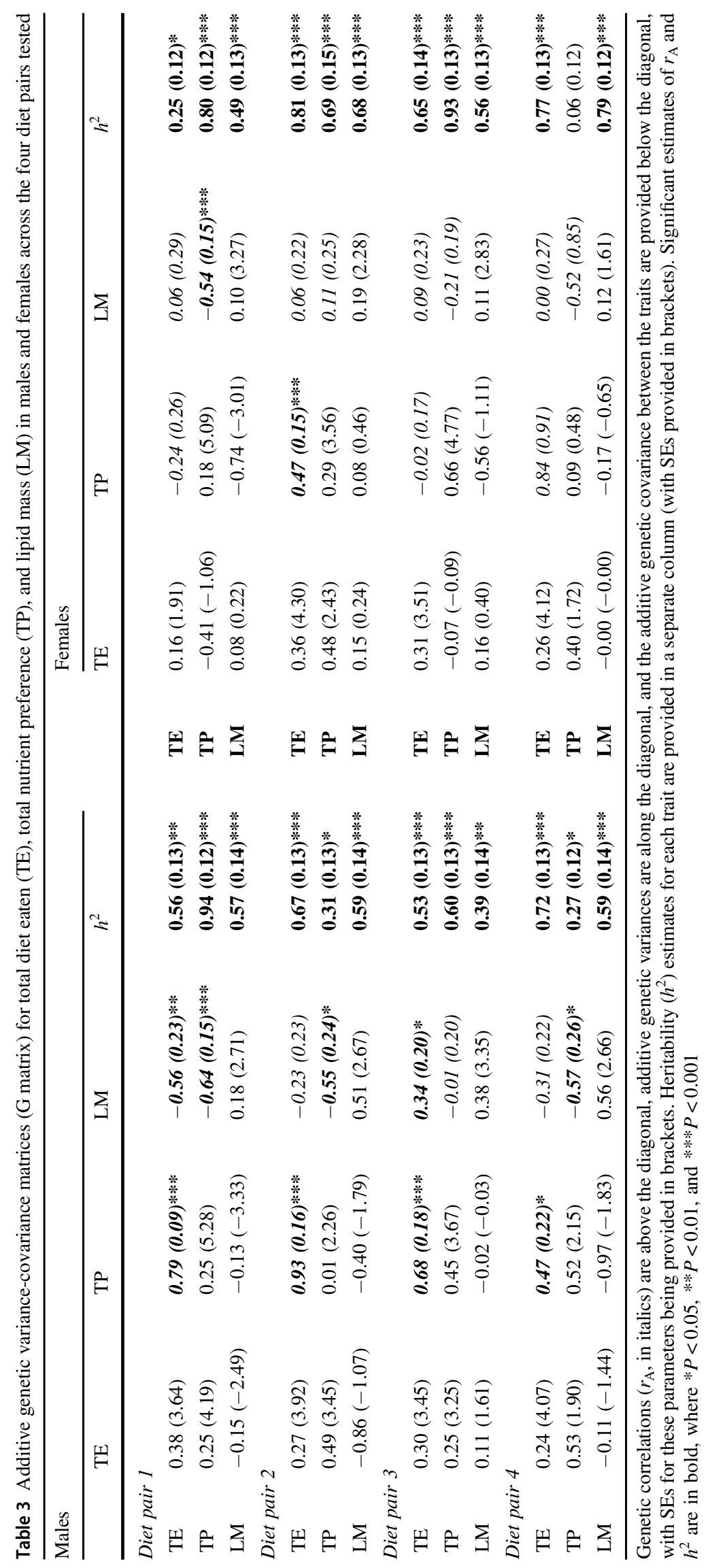


biased in females ( $\mathrm{P}: \mathrm{C}=1: 1.71)$ than males $(\mathrm{P}: \mathrm{C}=1: 2.02)$. This contrasts earlier work in T. commodus (Maklakov et al. 2008), the cockroach $N$. cinerea (Bunning et al. 2016), and D. melanogaster (Jensen et al. 2015) that showed the sexes regulate their intake of $\mathrm{P}$ and $\mathrm{C}$ in the same way, but is in agreement with work on the caterpillar Spodoptera litura (Lee 2010) and the cricket G. veletis (Harrison et al. 2014). In their study, Maklakov et al. (2008) argued that with both male and female T. commodus regulating their intake of $\mathrm{P}$ and $\mathrm{C}$ in the same way, there was evidence of intra-locus sexual conflict preventing either sex from regulating their nutrient intake towards their respective sex-specific dietary optima. The contrasting findings between these two studies of the same species might indicate that intra-locus sexual conflict has been resolved in our study population. However, this appears to be only a partial resolution, as our previous work with this population of $T$. commodus found that nutrient regulation did not coincide complete with nutritional optima for lifespan or reproductive effort in either sex (Rapkin et al. 2017). The sex differences in the feeding behaviour of $T$. commodus that we observe is likely to reflect the divergent reproductive strategies of the sexes. Reproductive success in female T. commodus is determined by the number of eggs produced, whereas male reproductive success in this species is largely determined by the amount of time spent calling to attract a mate (Bentsen et al. 2006). Although calling elevates metabolic rate (Kavanagh 1987), it is generally accepted that the energetic demands of egg production are higher (Trivers 1972), which explains the more pronounced compensatory feeding observed in females. In T. commodus, females require a relatively higher intake of $\mathrm{P}$ than males to maximize egg production ( $\mathrm{P}: \mathrm{C}=$ $1: 1)$, whereas males require a relatively higher intake of $\mathrm{C}$ than females to maximize calling effort $(\mathrm{P}: \mathrm{C}=1: 8)$ (Maklakov et al. 2008; Rapkin et al. 2017). The differences between our findings and those of previous studies clearly illustrates that the existence and direction of sex differences in feeding behaviour exhibits considerable variation across species. It is possible that this variation reflects species differences in the form of reproductive effort used by the sexes (e.g., calling, pheromone production, egg laying) and how energetically demanding they are, but this could be confirmed through future comparative studies on species that use a range of alternate reproductive strategies that vary in energetic and nutrient demands.

Current theories on the link between diet and obesity have highlighted the over ingestion of energy dense foods as a primary factor in weight gain (Mathes et al. 2011; Raubenheimer et al. 2015). While we cannot show "overingestion" in our study, we do show that lipid deposition in male and female $T$. commodus was significantly greater on the DP with the highest total nutrition (DP4) and lowest on the DP containing lowest total nutrition (DP1). However, we also show that lipid deposition is not only contingent on the energy (caloric) content of the diet but also the relative intake of nutrients. This is illustrated by the difference in lipid deposition of both sexes when feeding from DP2 and DP3; both DPs contain the same total energy content, but the highest nutrient diet in DP2 is C biased whereas it is P biased on DP3. Consequently, the significantly higher lipid deposition of males and females feeding from DP2 than DP3 suggests that the intake of C is more important to lipid deposition than $\mathrm{P}$ intake. Our response surface analysis also shows that $\mathrm{LM}$ was maximized in both sexes at a high intake of $\mathrm{C}$ and low intake of $\mathrm{P}$ (Table 2, Fig. 2). This finding supports the well-established link between increased C intake and lipid deposition reported in a range of animal taxa (Mathes et al. 2011; Raubenheimer et al. 2015). It also explains the lower LM of females than males on each of the DPs, despite their higher overall consumption of diets: by consuming relatively more $\mathrm{P}$ to $\mathrm{C}$ than males, female deposit fewer lipids. However, we cannot rule out other mechanisms that may explain the reduced LM in females, such as production of costly eggs. In insects, egg production requires a high intake of dietary P (e.g., Lee et al. 2008; Maklakov et al. 2008; Jensen et al. 2015) and a substantial mobilization of lipid reserves from the fat body to the ovaries in insects (Ziegler and Van Antwerpen 2006). It is therefore possible that the higher relative consumption of $\mathrm{P}$ promotes egg production and the greater utilization of lipid stores, resulting in lower LM in females than males. Unfortunately, our measure of LM measured the total lipids present in the entire body so we are unable to determine how lipids were allocated to specific organs or tissues. Future studies would benefit from a more specific measure of lipid deposition, as has been highlighted in studies examining the allocation of lipids to somatic and reproductive organs in female crickets (Gryllus firmus) with alternate dispersal morphs (the presence or absence of flight wings) (Zera 2005). An alternative solution would be to measure the LM of virgin females on each of the DPs, where egg production is substantially reduced (Nestel et al. 2005).

The physiological systems that control lipid deposition rely on a highly complex, polygenic contribution of genes. There are numerous examples of this from studies on mice (Marie et al. 2000; Cheverud et al. 2011) and humans (Raubenheimer et al. 2015; Robbins and Savage 2015), as well as a range of insect species (e.g., Horne et al. 2009; Schilder et al. 2011; Nanoth Vellichirammal et al. 2014). There is also a large number of studies showing that lipid deposition is influenced by the interaction between many variables, such as the dietary and social environment (Qi and Cho 2008; Mathes et al. 2011), microbiota (Schilder and Marden 2006; Wolf and Lorenz 2012), different life-history traits (Hansen et al. 2013), genes related to feeding behaviour and lipid deposition (e.g., "thrifty gene hypothesis") (Neel 1962; Barsh et al. 2000), and/ 
or different key genetic pathways (e.g., IGF-1, Post and Tatar 2016; mTOR, Kapahi et al. 2010; NHR-80, Goudeau et al. 2011). Our results are in broad agreement with the general view that lipid deposition is a complex trait that is influenced by the interaction between many variables. We show that LM in $T$. commodus is influenced by a complex interaction between genotype, the nutritional environment, and sex. Furthermore, there is considerable additive genetic covariance between LM, TE, and TP with the latter two feeding behaviours also subject to complex G-by-DP-by-Sex interactions. These findings demonstrate that to understand lipid deposition in T. commodus, it is not simply enough to characterize the independent contributions of the genotype, nutritional environment, and sex to this trait: context is important. These complex interactions mean that whether an individual is predisposed to increased lipid deposition cannot be predicted with any accuracy from any one of these variables in isolation. This finding directly challenges the "one size fits all" approach to weight loss by showing that any dietary intervention will be most effective when personalized to an individual's genotype, sex, and the dietary environment that they occupy (Qi and Cho 2008; Ordovas 2008).

Our results show an abundance of additive genetic variance for TE, TP, and LM, as well as a number of genetic correlations between these traits. This suggests that the control of an individual's nutrient intake and lipid deposition have a genetic basis but are also genetically linked, and therefore unlikely to evolve independently (Lande 1980). There are a number of consistent patterns in our genetic data. First, the number of significant genetic correlations, however, between TE, TP, and LM was greater in males than females (9 vs. 2). However, $h^{2}$ estimates were large for all traits and there were no systematic differences in these estimates across the sexes, indicating that this pattern is not due to a simple lack of additive genetic variance for these traits in females. This suggests that either the genetic pathway regulating feeding behaviour and LM is different in the sexes or it is the same but more tightly regulated in males than females. This may occur if nutrient intake or lipid storage is a more important determinant of fitness in males than females, for example, through their effects on regulating calling effort. Second, there were consistent positive genetic correlations between TE and TP across all DPs in males and also in DP2 for females. In our study, higher values of TP mean a stronger preference for P relative to $\mathrm{C}$, so that the positive genetic correlation between TE and TP indicates that in males and on some DPs for females the genes that govern the preference for $\mathrm{P}$ relative to $\mathrm{C}$ are positively associated with the genes for total dietary consumption. This genetic association may reflect the strength of the protein appetite system in this species or individual variation of male and female genotypes in how efficient they are at utilizing protein. Finally, there were negative genetic correlations between TP and LM on DP1, 3, and 4 in males and on DP1 in females, indicating that the genes for LM are negatively associated with those governing preference for $\mathrm{P}$ relative to $\mathrm{C}$. Both of these patterns of additive genetic covariance between traits provide partial support for the PLH at the genetic level. The PLH predicts that in a nutritionally imbalanced environment where $\mathrm{P}$ is limited, the powerful $\mathrm{P}$ appetite will stimulate individuals to increase their dietary consumption in an attempt to gain more P (e.g., Simpson and Raubenheimer 2005), a pattern that is supported by the positive genetic correlation between $\mathrm{TP}$ and TE. For example, there is a genetic correlation of $0.93( \pm 0.16)$ between TE and TP for males on DP2. As DP2 is highly $\mathrm{C}$ biased, males increase their $\mathrm{P}$ intake by consuming more of the available diets. A side effect of the PLH is the overingestion of more abundant nutrients (such as C), resulting in increased lipid deposition and predisposing of an individual to obesity (Simpson and Raubenheimer 2005), which is consistent with the observed negative genetic correlations between TP and LM. However, this negative genetic correlation could also support the alternate view that the genes for absolute $\mathrm{C}$ preference are directly linked to those for LM, although evidence for this in our study is currently weak.

In conclusion, while our work is in general agreement with the commonly held view that the consumption of energy-rich diets is a major contributor to the increased rates of obesity observed in most developed societies, it also clearly demonstrates that the causes of increased lipid deposition are far more complex than this, at least in $T$. commodus. Complex interactions between genotype, the nutritional environment, and sex for feeding behaviour (TE and TP) and LM, as well as additive genetic covariance between these traits, means that focussing on any one of these variables in isolation will provide an incomplete understanding on whether an individual is predisposed to lipid deposition (or obesity) or not. The obvious question that remains from our work is what are the consequences of increased lipid deposition in male and female $T$. commodus? In humans, as well as a range of mammalian models, there is clear evidence that excessive lipid deposition and obesity are responsible for a range of metabolic and cardiovascular disorders (Raubenheimer et al. 2015), which negatively impact health. There is also growing evidence that similar disorders exist in insects (e.g., D. melanogaster, Musselman et al. 2011; Libeullula pulchella, Schilder and Marden 2006) and that there are fitness costs of obesity (e.g., D. melanogaster, Skorupa et al. 2008; Musselman et al. 2011; $\mathrm{Na}$ et al. 2013; L. pulchella, Schilder and Marden 2006; Plutella xylostella, Warbrick-Smith et al. 2006), which supports the general suitability of using insects as models in obesity research. We do not currently know, however, if similar physiological disorders and fitness costs of obesity 
exist in T. commodus or if the degree of lipid deposition we observe in our study only has a beneficial role in this species. For example, lipid deposition and the resulting lipid metabolism is known to be essential for growth and reproduction in a range of insects, as well as providing energy needed during extended non-feeding periods (e.g., dispersal) (Arrese and Soulages 2010). Clearly, more studies are needed that clarify the fitness costs and benefits of lipid deposition in male and female $T$. commodus.

\section{Data accessibility}

Data available from the Dryad Digital Repository: https:// doi.org/10.5061/dryad.cj3315s.

Acknowledgements JH was funded by a University Royal Society Fellowship and Equipment Grant and by NERC (NE/G00949X/1) and AJW by a BBSRC Fellowship. JR was funded by a NERC studentship (NERC/1200242) awarded to JH.

\section{Compliance with ethical standards}

Conflict of interest The authors declare that they have no conflict of interest.

\section{References}

Arrese EL, Soulages JL (2010) Insect fat body: energy, metabolism, and regulation. Annu Rev Entomol 55:207-225

Barreto RE, Moreira PSA, Carvalho RF (2003) Sex-specific compensatory growth in food-deprived Nile tilapia. Braz J Med Biol Res 36:477-483

Barsh GS, Farooqi IS, O'Rahilly S (2000) Genetics of body-weight regulation. Nature 404:644-651

Bentsen CL, Hunt J, Jennions MD, Brooks R (2006) Complex multivariate sexual selection on male acoustic signaling in a wild population of Teleogryllus commodus. Am Nat 167:E102-E116

Brooks RC, Simpson SJ, Raubenheimer D (2010) The price of protein: combining evolutionary and economic analysis to understand excessive energy consumption. Obes Rev 11:887-894

Bunning H, Bassett L, Clowser C, Rapkin J, Jensen K, House CM, Archer CR, Hunt J (2016) Dietary choice for a balanced nutrient intake increases the mean and reduces the variance in the reproductive performance of male and female cockroaches Ecol Evol 6:4711-4730

Bunning H, Rapkin J, Belcher L, Archer CR, Jensen K, Hunt J (2015) Protein and carbohydrate intake influence sperm number and fertility in male cockroaches, but not sperm viability. Proc R Soc B Biol Sci 282:20142144

Cheverud JM, Lawson HA, Fawcett GL, Wang B, Pletscher LS, Fox $\mathrm{AR}$ et al. (2011) Diet-dependent genetic and genomic imprinting effects on obesity in mice. Obesity 19:160-170

Coatmellec-Taglioni G, Dausse J-P, Giudicelli Y, Ribière C (2003) Sexual dimorphism in cafeteria diet-induced hypertension is associated with gender-related difference in renal leptin receptor down-regulation. J Pharmacol Exp Ther 305:362-367

Faith MS, Rha SS, Neale MC, Allison DB (1999) Evidence for genetic influences on human energy intake: results from a twin study using measured observations. Behav Genet 29:145-154
Felton AM, Felton A, Raubenheimer D, Simpson SJ, Foley WJ, Wood JT et al. (2009) Protein content of diets dictates the daily energy intake of a free-ranging primate. Behav Ecol 20:685-690

Gilmour AR, Gogel BJ, Cullis BR, Thompson R, Butler D (2009) ASReml user guide release 3.0. VSN Int. Ltd., Hemel Hempstead

Gordon RR, Hunter KW, Sørensen P, Pomp D (2008) Genotypex diet interactions in mice predisposed to mammary cancer. I. Body weight and fat Mamm Genome 19:163-178

Gosby AK, Conigrave AD, Lau NS, Iglesias MA, Hall RM, Jebb SA et al. (2011) Testing protein leverage in lean humans: a randomised controlled experimental study. PLoS ONE 6:e25929

Gosby AK, Conigrave AD, Raubenheimer D, Simpson SJ (2014) Protein leverage and energy intake. Obes Rev 15:183-191

Goudeau J, Bellemin S, Toselli-Mollereau E, Shamalnasab M, Chen Y, Aguilaniu H (2011) Fatty acid desaturation links germ cell loss to longevity through NHR-80/HNF4 in C. elegans. PLoS Biol 9: e1000599

Hansen M, Flatt T, Aguilaniu H (2013) Reproduction, fat metabolism, and life span: what is the connection? Cell Metab 17:10-19

Harrison S, Raubenheimer D, Simpson S, Godin G, Bertram S (2014) Towards a synthesis of frameworks in nutritional ecology: interacting effects of protein, carbohydrate and phosphorus on field cricket fitness. Proc R Soc B Biol Sci 281:20140539

Hasselbalch AL, Heitmann BL, Kyvik KO, Sørensen TIA (2010) Associations between dietary intake and body fat independent of genetic and familial environmental background. Int J Obes 34:892

Heianza Y, Qi L (2017) Gene-diet interaction and precision nutrition in obesity. Int J Mol Sci 18:787

Henson SM, Hallam TG (1995) Optimal feeding via constrained processes. J Theor Biol 176:33-37

Horne I, Haritos VS, Oakeshott JG (2009) Comparative and functional genomics of lipases in holometabolous insects. Insect Biochem Mol Biol 39:547-567

Jensen K, McClure C, Priest NK, Hunt J (2015) Sex-specific effects of protein and carbohydrate intake on reproduction but not lifespan in Drosophila melanogaster. Aging Cell 14:605-615

Kanter R, Caballero B (2012) Global gender disparities in obesity: a review. Adv Nutr 3:491-498

Kapahi P, Chen D, Rogers AN, Katewa SD, Li PW-L, Thomas EL et al. (2010) With TOR, less is more: a key role for the conserved nutrient-sensing TOR pathway in aging. Cell Metab 11:453-465

Kavanagh MW (1987) The efficiency of sound production in two cricket species, Gryllotalpa australis and Teleogryllus commodus (Orthoptera: Grylloidea). J Exp Biol 130:107-119

van der Klaauw AA, Farooqi IS (2015) The hunger genes: pathways to obesity. Cell 161:119-132

Klimentidis YC, Beasley TM, Lin H-Y, Murati G, Glass GE, Guyton $\mathrm{M}$ et al. (2011) Canaries in the coal mine: a cross-species analysis of the plurality of obesity epidemics. Proc R Soc B Biol Sci 278:1626-1632

Lande R (1979) Quantitative genetic analysis of multivariate evolution, applied to brain: body size allometry. Evolution 33:402-416

Lande R (1980) Sexual dimorphism, sexual selection, and adaptation in polygenic characters. Evolution 34:292-305

Lee KP (2010) Sex-specific differences in nutrient regulation in a capital breeding caterpillar, Spodoptera litura (Fabricius). J Insect Physiol 56:1685-1695

Lee KP, Simpson SJ, Clissold FJ, Brooks R, Ballard JW, Taylor PW et al. (2008) Lifespan and reproduction in Drosophila: new insights from nutritional geometry. Proc Natl Acad Sci USA 105:2498-2503

Lihoreau M, Buhl J, Charleston MA, Sword GA, Raubenheimer D, Simpson SJ (2015) Nutritional ecology beyond the individual: a conceptual framework for integrating nutrition and social interactions. Ecol Lett 18:273-286 
Link JC, Reue K (2017) Genetic basis for sex differences in obesity and lipid metabolism. Annu Rev Nutr 37:225-245

Liu J, Lloyd SG (2013) High-fat, low-carbohydrate diet alters myocardial oxidative stress and impairs recovery of cardiac function after ischemia and reperfusion in obese rats. Nutr Res 33:311-321

Maklakov AA, Simpson SJ, Zajitschek F, Hall MD, Dessmann J, Clissold F et al. (2008) Sex-specific fitness effects of nutrient intake on reproduction and lifespan. Curr Biol 18:1062-1066

Marie LS, Miura GI, Marsh DJ, Yagaloff K, Palmiter RD (2000) A metabolic defect promotes obesity in mice lacking melanocortin4 receptors. Proc Natl Acad Sci USA 97:12339-12344

Martens EA, Lemmens SG, Westerterp-Plantenga MS (2013) Protein leverage affects energy intake of high-protein diets in humans. Am J Clin Nutr 97:86-93

Mathes WF, Kelly SA, Pomp D (2011) Advances in comparative genetics: influence of genetics on obesity. Br J Nutr 106:S1-S10

Medrikova D, Jilkova ZM, Bardova K, Janovska P, Rossmeisl M, Kopecky J (2012) Sex differences during the course of dietinduced obesity in mice: adipose tissue expandability and glycemic control. Int J Obes 36:262

Musselman LP, Fink JL, Narzinski K, Ramachandran PV, Hathiramani SS, Cagan RL et al. (2011) A high-sugar diet produces obesity and insulin resistance in wild-type Drosophila. Dis Model Mech 4:842-849

Na J, Musselman LP, Pendse J, Baranski TJ, Bodmer R, Ocorr K et al. (2013) A Drosophila model of high sugar diet-induced cardiomyopathy. PLoS Genet 9:e1003175

Nanoth Vellichirammal N, Zera AJ, Schilder RJ, Wehrkamp C, Riethoven J-JM, Brisson JA (2014) De novo transcriptome assembly from fat body and flight muscles transcripts to identify morphspecific gene expression profiles in Gryllus firmus. PLoS ONE 9: e82129

Neel JV (1962) Diabetes mellitus: a 'thrifty' genotype rendered detrimental by 'progress'? Am J Hum Genet 14:353-362

Nestel D, Papadopoulos NT, Liedo P, Gonzales-Ceron L, Carey JR (2005) Trends in lipid and protein contents during medfly aging: an harmonic path to death. Arch Insect Biochem Physiol 60:130-139

Ordovas JM (2008) Genotype-phenotype associations: modulation by diet and obesity. Obesity 16:S40-S46

Post S, Tatar M (2016) Nutritional geometric profiles of insulin/IGF expression in Drosophila melanogaster. PLoS ONE 11:e0155628

Qi L, Cho YA (2008) Gene-environment interaction and obesity. Nutr Rev 66:684-694

Rapkin J, Archer CR, Grant CE, Jensen K, House CM, Wilson AJ et al. (2017) Little evidence for intralocus sexual conflict over the optimal intake of nutrients for life span and reproduction in the black field cricket Teleogryllus commodus. Evolution 77:2159-2177

Rapkin J, Jensen K, Archer CR, House CM, Sakaluk SK, Castillo ED, Hunt J (2018) The Geometry of Nutrient Space-Based LifeHistory Trade-Offs: Sex-Specific Effects of Macronutrient Intake on the Trade-Off between Encapsulation Ability and Reproductive Effort in Decorated Crickets Am Nat 191:452-474

Raubenheimer D, Machovsky-Capuska GE, Gosby AK, Simpson SJ (2015) Nutritional ecology of obesity: from humans to companion animals. Br J Nutr 113:S26-S39

Raubenheimer D, Simpson SJ (1997) Integrative models of nutrient balancing: application to insects and vertebrates. Nutr Res Rev 10:151-179

Reddiex AJ, Gosden TP, Bonduriansky R, Chenoweth SF (2013) Sexspecific fitness consequences of nutrient intake and the evolvability of diet preferences Am Nat 182:91-102

Reed LK, Williams S, Springston M, Brown J, Freeman K, DesRoches CE, Sokolowski MB, Gibson G (2010) Genotype-by-diet interactions drive metabolic phenotype variation in Drosophila melanogaster. Genetics 185:1009-1019

Robbins AL, Savage DB (2015) The genetics of lipid storage and human lipodystrophies. Trends Mol Med 21:433-438

Schilder RJ, Marden JH (2006) Metabolic syndrome and obesity in an insect. Proc Natl Acad Sci USA 103:18805-18809

Schilder RJ, Zera AJ, Black C, Hoidal M, Wehrkamp C (2011) The biochemical basis of life history adaptation: molecular and enzymological causes of NADP+-isocitrate dehydrogenase activity differences between morphs of Gryllus firmus that differ in lipid biosynthesis and life history. Mol Biol Evol 28:3381-3393

Simpson SJ, Raubenheimer D (2005) Obesity: the protein leverage hypothesis. Obes Rev 6:133-142

Simpson SJ, Raubenheimer D (2012) The nature of nutrition: a unifying framework from animal adaptation to human obesity. Princeton University Press, Princeton, NJ

Skorupa DA, Dervisefendic A, Zwiener J, Pletcher SD (2008) Dietary composition specifies consumption, obesity and lifespan in Drosophila melanogaster. Aging Cell 7:478-490

Smith BK, Andrews PK, West DB (2000) Macronutrient diet selection in thirteen mouse strains. Am J Physiol Integr Comp Physiol 278: R797-R805

Song Y, Wang H-J, Dong B, Ma J, Wang Z, Agardh A (2016) 25-year trends in gender disparity for obesity and overweight by using WHO and IOTF definitions among Chinese school-aged children: a multiple cross-sectional study. BMJ Open 6:e011904

Sørensen A, Mayntz D, Raubenheimer D, Simpson SJ (2008) Proteinleverage in mice: the geometry of macronutrient balancing and consequences for fat deposition. Obesity 16:566-571

South SH, House CM, Moore AJ, Simpson SJ, Hunt J (2011) Male cockroaches prefer a high carbohydrate diet that makes them more attractive to females: implications for the study of condition dependence. Evolution 65:1594-1606

Stephens DW, Krebs JR (1986) Foraging theory. Princeton University Press, Princeton, NJ

Sutton GM, Trevaskis JL, Hulver MW, McMillan RP, Markward NJ, Babin MJ, Meyer EA, Butler AA (2006) Diet-genotype interactions in the development of the obese, insulin-resistant phenotype of C57BL/6J micelacking melanocortin-3 or-4 receptors Endocrinology 147:2183-2196

Tanaka T (2014) Genetics of energy and macronutrient intake in humans. Curr Nutr Rep 3:170-177

Trivers R (1972) Parental investment and sexual selection. In: Campbell B (ed) Sexual selection and the descent of man 18711971. Aldine Publishing Company, Chicago, IL, pp 136-179

Warbrick-Smith J, Behmer ST, Lee KP, Raubenheimer D, Simpson SJ (2006) Evolving resistance to obesity in an insect. Proc Natl Acad Sci USA 103:14045-14049

Wilson AJ, Reale D, Clements MN, Morrissey MM, Postma E, Walling CA et al. (2010) An ecologist's guide to the animal model. J Anim Ecol 79:13-26

Wolf KJ, Lorenz RG (2012) Gut microbiota and obesity. Curr Obes Rep 1:1-8

Yuan C, Gaskins AJ, Blaine AI, Zhang C, Gillman MW, Missmer SA et al. (2016) Association between cesarean birth and risk of obesity in offspring in childhood, adolescence, and early adulthood. JAMA Pediatr 170:e162385-e162385

Zera AJ (2005) Intermediary metabolism and life history trade-offs: lipid metabolism in lines of the wing-polymorphic cricket, Gryllus firmus, selected for flight capability vs. early age reproduction. Integr Comp Biol 45:511-524

Ziegler R, Van Antwerpen R (2006) Lipid uptake by insect oocytes. Insect Biochem Mol Biol 36:264z-272 